\title{
Treatment Pulse Application for Magnetic Stimulation
}

\author{
Sun-Seob Choi and Whi-Young Kim \\ Neuroradiology Section, Department of Radiology, Dong-A University Medical Center, 3Ga-1, Dongdaeshin-Dong, Seo-Gu, \\ Busan 602-715, Republic of Korea
}

Correspondence should be addressed to Whi-Young Kim,ndyag@dongju.ac.kr

Received 14 November 2010; Accepted 11 February 2011

Academic Editor: Mohammed Rachidi

Copyright ( $) 2011$ S.-S. Choi and W.-Y. Kim. This is an open access article distributed under the Creative Commons Attribution License, which permits unrestricted use, distribution, and reproduction in any medium, provided the original work is properly cited.

\begin{abstract}
Treatment and diagnosis can be made in difficult areas simply by changing the output pulse form of the magnetic stimulation device. However, there is a limitation in the range of treatments and diagnoses of a conventional sinusoidal stimulation treatment pulse because the intensity, width, and form of the pulse must be changed according to the lesion type. This paper reports a multidischarge method, where the stimulation coils were driven in sequence via multiple switching control. The limitation of the existing simple sinusoidal pulse form could be overcome by changing the intensity, width, and form of the pulse. In this study, a new sequential discharge method was proposed to freely alter the pulse width. The output characteristics of the stimulation treatment pulse were examined according to the trigger signal delay applied to the switch at each stage by applying a range of superposition pulses to the magnetic simulation device, which is widely used in industry and medicine.
\end{abstract}

\section{Introduction}

The variable pulse of the magnetic stimulation device enables a diagnosis of various diseases that are difficult to diagnose or treat $[1,2]$. The human body absorbs the magnetic pulse of the magnetic stimulation device and forms a local eddy current [3-5]. The temperature increase varies according to the treatment or diagnostic processes because the formation of an eddy current depends on the magnetic pulse intensity and human immunological characteristics $[2,6,7]$. Therefore, more sophisticated treatment and diagnosis can be realized by appropriately controlling the pulse intensity and subsequent increase in temperature [3].

The existing sinusoidal-stimulation treatment pulse forms are unable to appropriately address diverse treatments and diagnoses because different intensities, pulse widths, and pulse forms are needed for each treatment and diagnosis [8]. The magnetic stimulation device has a large stimulation coil capacity because the stimulation frequency must vary according to its purpose. It forms a magnetic field in pulse form and generates an eddy current in the human body [9]. This performance requires a power supply, high-voltage resistance, high repetition, high-speed switching element, and wide range of control techniques [10]. Therefore, the limitation of the treatment and diagnosis based on a sinusoidal pulse form can be overcome by controlling the pulse intensity, width, and form of the magnetic stimulation device where necessary.

This paper proposes a new sequential discharge method to freely change the pulse width. In this method, the stimulation coils were driven sequentially by the control circuit. The AT80S8535 one-chip microprocessor technology of the AVR system and PFN were combined to actively superpose one, two, three, and four-stage circuits and produce high-voltage pulse forms with diverse pulse widths.

\section{Design}

2.1. Composition of the Magnetic Stimulation Device. Figure 1 shows a schematic diagram of the magneticstimulation device, which consists of the stimulation coil part, power supply, and controller. The long cylindrical form was wound with a coil in a loop type to efficiently deliver the current pulse discharged from the capacitor. Continuous operation of the stimulation device can result in heating of the refrigerant in the stimulation device and stimulation coil, as well as a spatial change in penetrability due to the effect on the overall temperature distribution. 


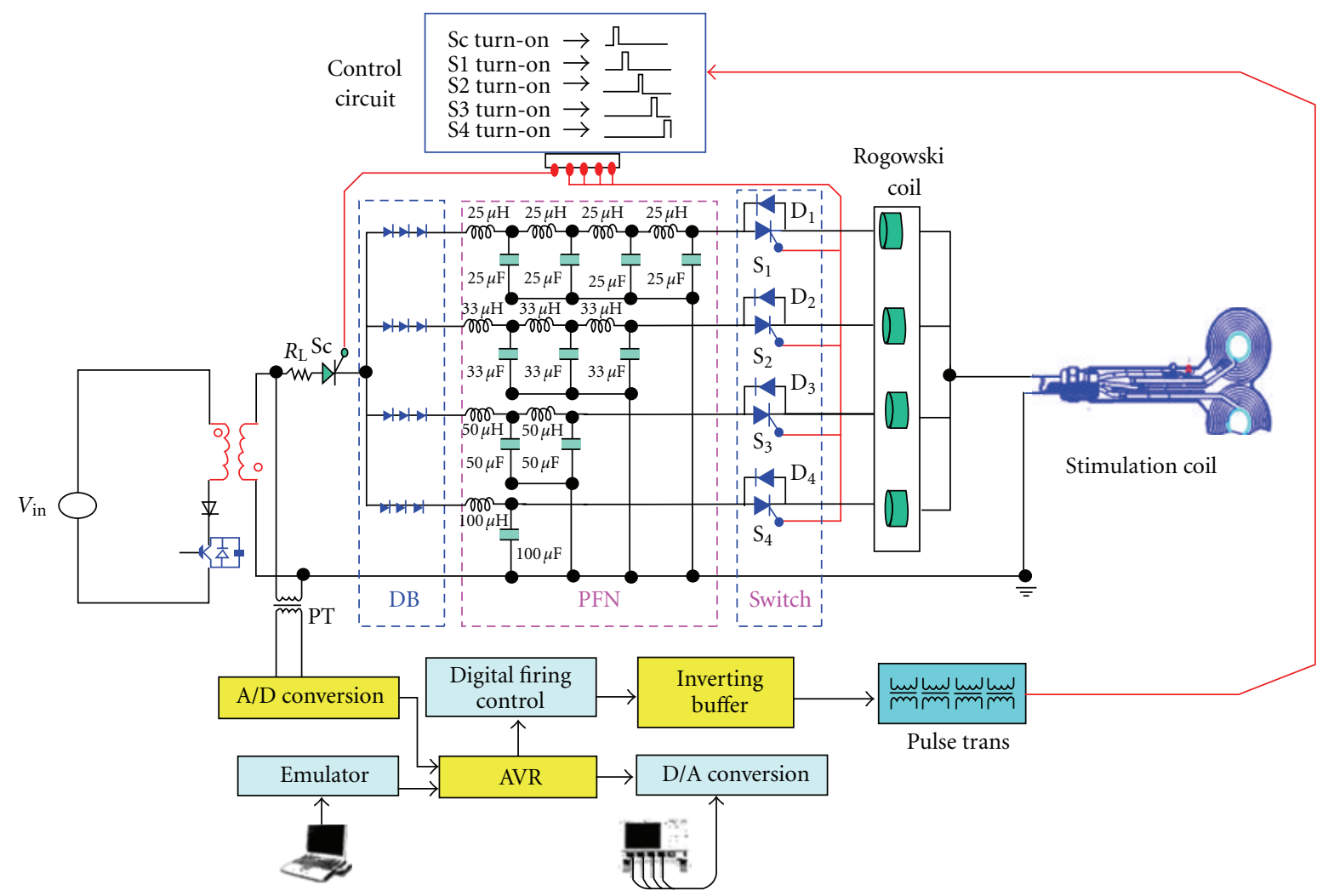

FIgURE 1: Diagram of the proposed magnetic stimulation device.

2.2. Power Supply. The most important part of the highvoltage flyback converter is that it has a high turn ratio between the primary and secondary sides to generate a high voltage. The high voltage and many turns of the secondary side cause parasitic capacitance. This parasitic capacitance generates parasitic resonance of the converter current and voltage under the transient state, increases the current stress in the switching device, and limits the converter switching frequency. Therefore, a soft-switching technique, zero-current switching (ZCS), was employed in this study to reduce the effect of parasitic capacitance of the secondary side. IRG4PH50UD and EI40 (TDK) were used as elements.

Most of the existing stimulation pulse forms for diagnosis and treatment are sinusoidal, which limits the range of treatments. This limitation in the treatment and diagnosis based on the sinusoidal pulse form can be overcome by controlling the pulse intensity, width, and form of the magnetic stimulation device where necessary. Multiple energy pulses in a magnetic stimulation device result in deeper penetration and produce better results than a single energy pulse at the same average output.

Figure 1 shows a sequential discharge control type stimulation device, to which diverse PFN methods were applied. When the SCR $S_{C}$ is turned on, the energy is charged in the capacitor of PFN. If S1, S2, S3, and S4 are turned on sequentially for meshes $1,2,3$, and 4, respectively, the device begins the operation. The energy stored in the capacitor of the PFN is transferred to the stimulation coil for its action. The stimulation coil can work in a range of ways as a single or multiple-mesh circuit. This circuit has the desired current pulse when the charged energy is transferred to the stimulation coil. Equation (1) shows the energy stored in the capacitance $\mathrm{C}$

$$
E_{O}=\frac{1}{2} C V_{O}^{2}
$$

The power consumption of a magnetic stimulation device can be expressed as

$$
P=\frac{f_{T}(E)}{\zeta}
$$

where $f_{T}$ is the pulse train frequency, $E$ is the energy dissipated per pulse, and $\zeta$ is the capacitor charger efficiency

$$
E=\frac{1}{2} \sum_{i=1}^{n} C V_{C i}^{2}(t=0)-\frac{1}{2} \sum_{i=1}^{n} C V_{C i}^{2}(t \geq t p) .
$$

It is assumed that the capacitor charger is turned off or contributes a negligible amount of charge during the pulse. The stimulating coil temperature is proportional to square of the coil current integrated over the pulse duration, which is sometimes called the load integral

$$
\operatorname{Stim}_{\text {coil }}=\propto \int_{0}^{t \geq t p} I^{2} d t=\int_{0}^{t p} I^{2} d t+\int_{t p}^{t \geq t p} I^{2} d t .
$$




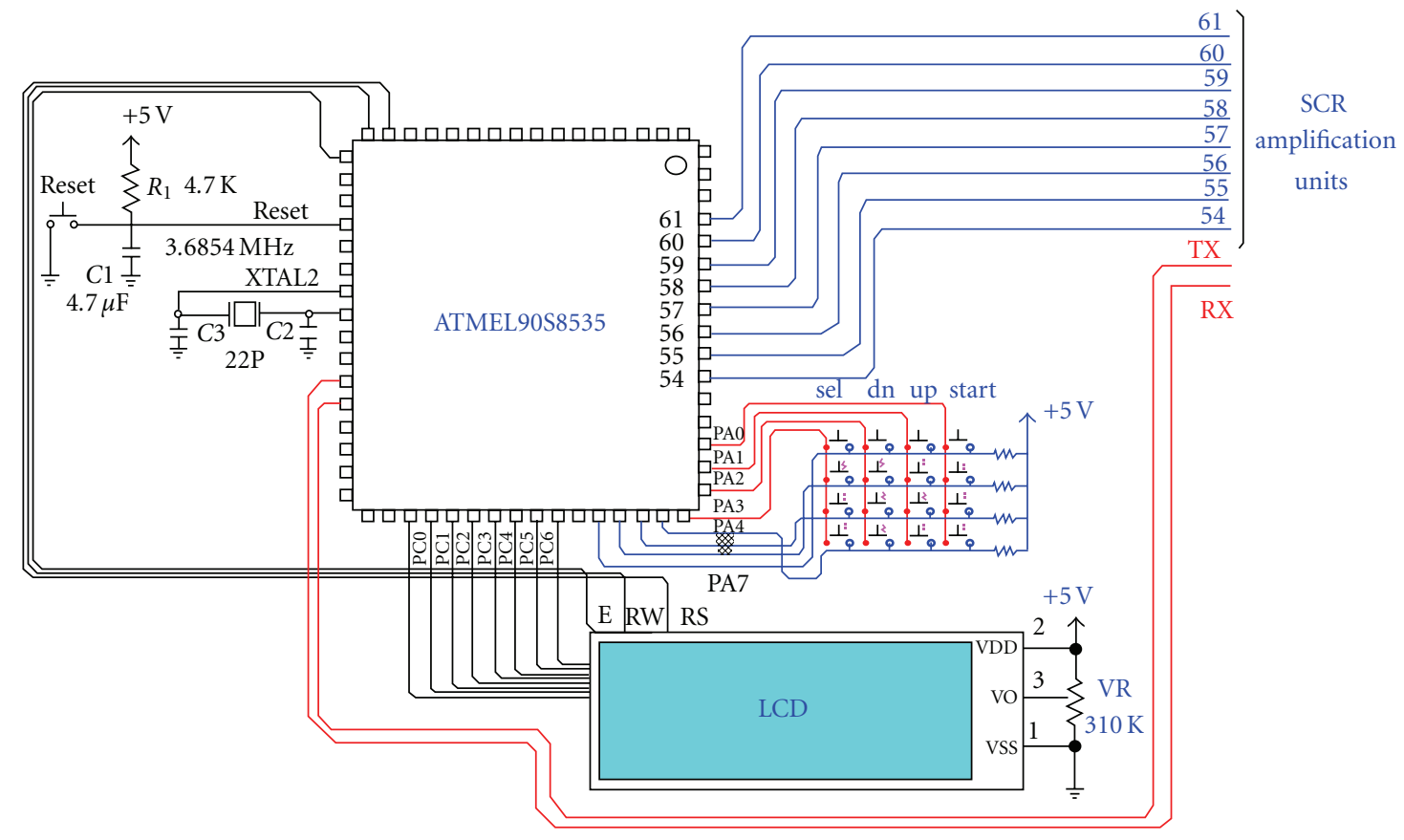

FIGURE 2: AVR one-chip control and drive circuit.

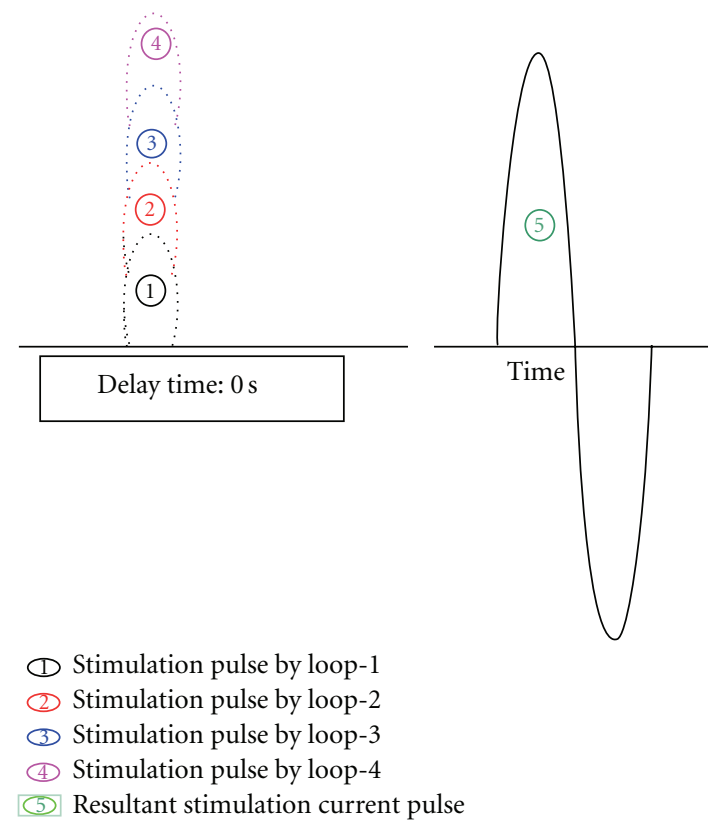

(a)

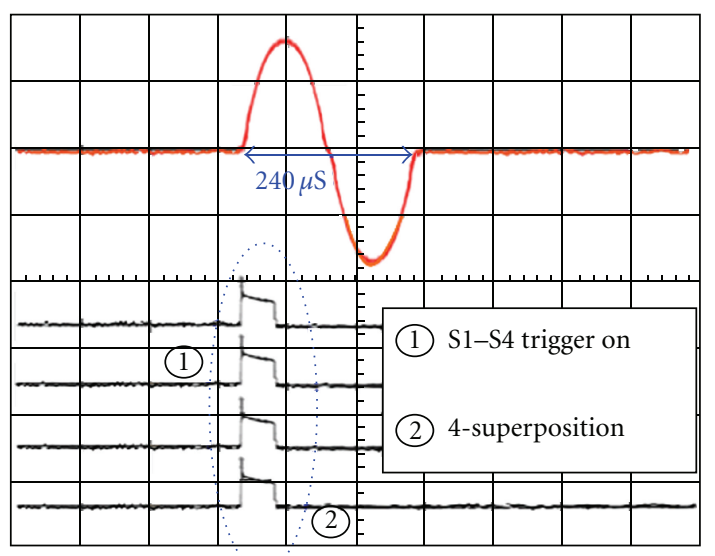

(b)

Figure 3: (a) Idea, (b) test. S1, S2, S3, S4: trigger on (delay: 0 s; pulse width: $240 \mu$ S).

A single-mesh PFN generates sinusoidal pulses but a multiple-mesh PFN generates square wave pulses with similar peak and average powers. Variable pulse forms can broaden the range of disease treatments. In this study, a new sequential discharge method was proposed to freely change the pulse width. The stimulation coils were driven sequentially by the control circuit. The AT80S8535 onechip microprocessor technology of the AVR system and PFN were combined to actively superpose $1 \sim 4$ stage circuits and to produce high-voltage pulse forms with a range of pulse widths. The power characteristics of the stimulation treatment pulse were examined according to the trigger signal delay applied to the switch at each stage by applying a wide range of superposition pulses to the magnetic stimulation device, which is used widely in industry and medicine. 


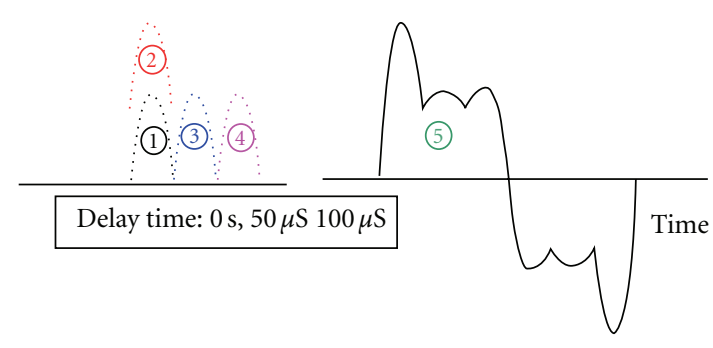

(1) Stimulation pulse by loop-1

(2) Stimulation pulse by loop-2

(3) Stimulation pulse by loop-3

(4) Stimulation pulse by loop-4

(5) Resultant stimulation current pulse

(a)

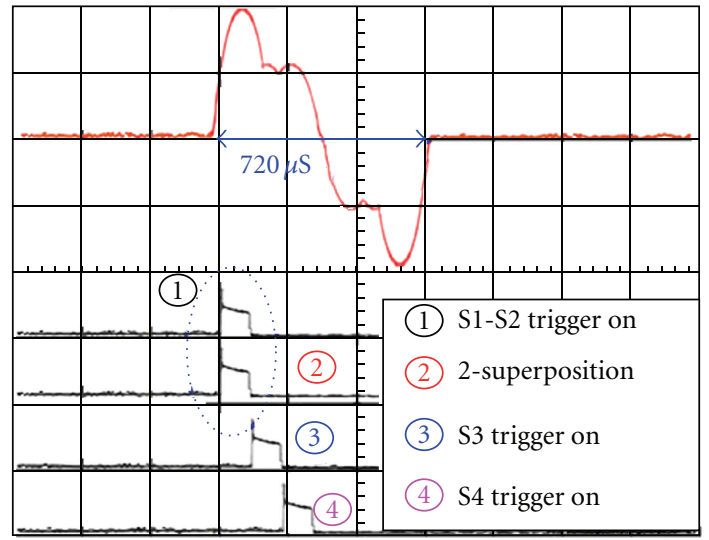

(b)

Figure 4: (a) Idea, (b) test. S1, S2 $\rightarrow$ S3 $\rightarrow$ S4: trigger on (delay: $0 \mathrm{~s}, 50 \mu \mathrm{S}, 100 \mu \mathrm{S}$; pulse width: $720 \mu \mathrm{S}$ ).

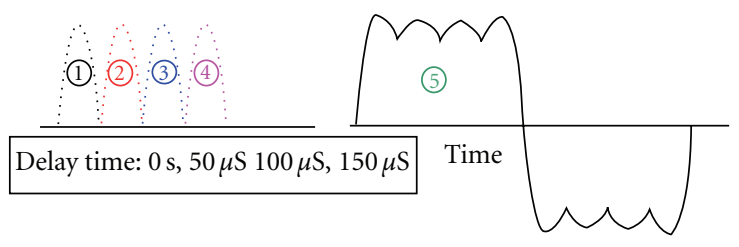

(1) Stimulation pulse by loop-1

(2) Stimulation pulse by loop-2

(3) Stimulation pulse by loop-3

(4) Stimulation pulse by loop-4

(5) Resultant stimulation current pulse

(a)

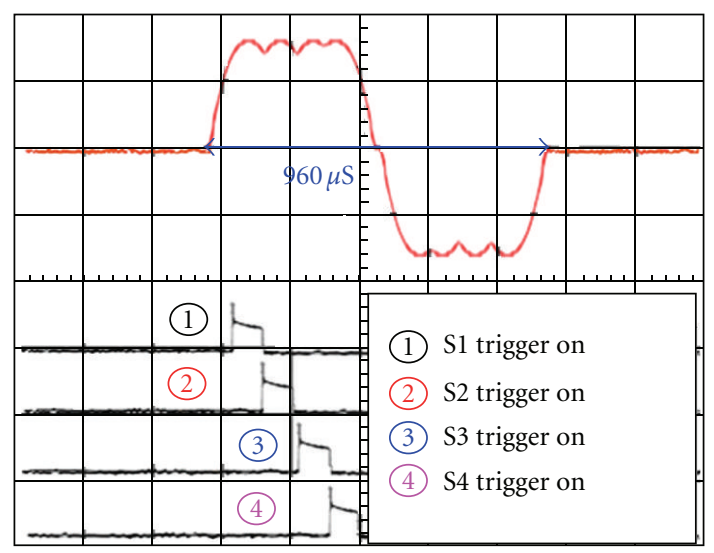

(b)

FIGURE 5: (a) Idea, (b) test. $\mathrm{S} 1 \rightarrow \mathrm{S} 2 \rightarrow \mathrm{S} 3 \rightarrow \mathrm{S} 4$ : trigger on (delay: $0 \mathrm{~s}$, $50 \mu \mathrm{S}, 100 \mu \mathrm{S}, 150 \mu \mathrm{S}$; pulse width: $960 \mu \mathrm{S})$.

2.3. Controller. Figure 2 shows an SCR time control circuit, which consists of an AVR one-chip microprocessor. The control circuit has four parts: (1) a keyboard that inputs the time control, (2) a LCD device that indicates the input time state, (3) an AVR microprocessor (ATMEL), which is the core of the control circuit, and (4) an amplification circuit that turns on the SCR. The control circuit operates as follows. The delay data inputted via the keyboard is transferred to the AVR, which outputs a range of signals according to the predefined program. One signal from the AVR is the LCD display signal, and the other is the trigger signal, which triggers the SCR in the pulse-power-switching circuit according to the desired delay time. The variable range for SCR1-SCR2, SCR2-SCR3, SCR3-SCR4, and SCR4-SCR5 was $0 \sim 500 \mu \mathrm{S}$ in $100 \mu \mathrm{S}$ increments. The amplified signal first turns on the SCRc then SCR1 SCR4, sequentially.

\section{Experiment Results}

The energy stored in the capacitor of each PFN circuit was applied to the stimulation coil through the switching of the SCR. The energy stored in the capacitor of each PFN circuit was applied sequentially with a specific delay. The current waveform of the stimulation coil was measured while changing the delay of the trigger conducting the angle of each SCR from 0 to $500 \mu \mathrm{S}$. The current and voltage were measured using a high-voltage probe of (Probe X 6,000 V, Lecroy) and a Rogowski current waveform transducer (CWT, Penmuk).

Assuming no loss from the resonance or discharge circuit, there will be no change in VC at the start and end of the discharge. On the other hand, a loss is generated due to the electric resistance of the magnetic field generation coil and wiring, which causes a decrease in discharge completion voltage. With this voltage drop, the power loss of the discharge circuit can be calculated using (5). However, the power capacity of the high-voltage power that charges the discharge capacitor must be greater than the power loss.

C was $100 \mu \mathrm{F}$ and $2000 \mathrm{~V}$, and L was $100 \mu \mathrm{H}$. The SCR for the discharge operation was a SEMIKRON SKT760 exclusive module, and parts with sufficient current capacity and withstand voltage were used for high-voltage, highcurrent circuit wiring. The initial discharge voltage $\mathrm{Vi}$ and discharge end voltage Ve were $1,000 \mathrm{~V}$ and $760 \mathrm{~V}$, respectively. Therefore, the power loss can be calculated using the following equation:

$$
P_{\text {loss }}=\frac{1}{2} C f\left[\left(\nu_{\text {ini }}\right)^{2}-\left(\nu_{\text {end }}\right)^{2}\right] .
$$




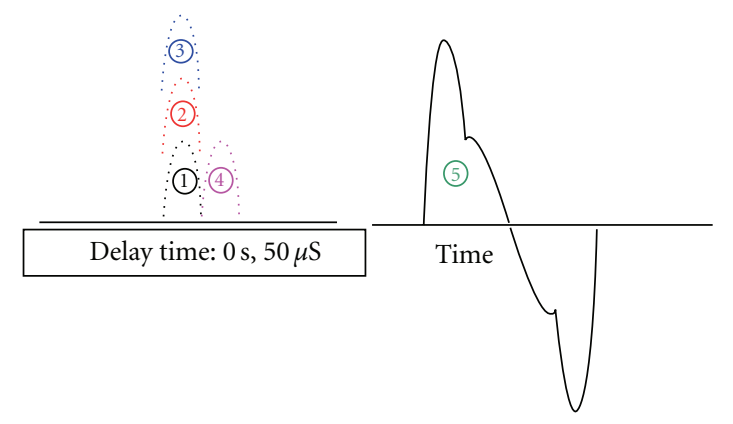

Stimulation pulse by loop-1

Stimulation pulse by loop-2

(3) Stimulation pulse by loop-3

(4) Stimulation pulse by loop-4

(5) Resultant stimulation current pulse

(a)

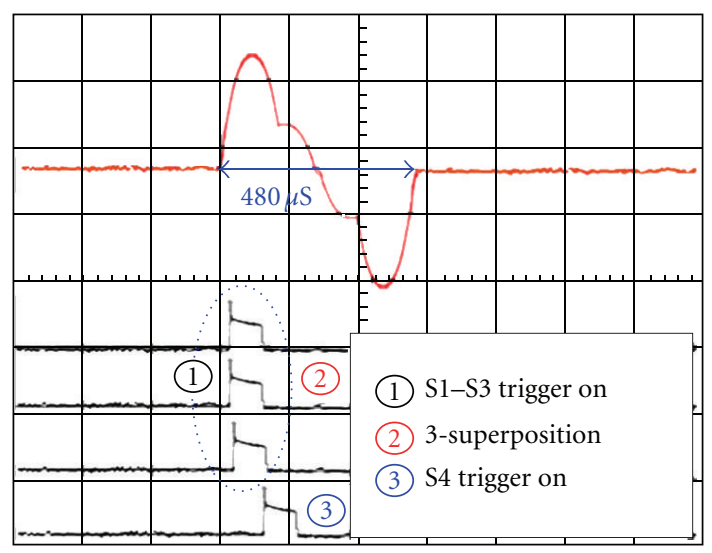

(b)

Figure 6: (a) Idea, (b) test. S1, S2, S3 $\rightarrow$ S4: trigger on (delay: 0 s, 50 $\mu \mathrm{S}$; pulse width: $480 \mu \mathrm{S})$.

When Vi was 1,000 V, a time-dependent magnetic field of $1 \mathrm{~T}$ appeared at a point $50 \mathrm{~mm}$ from the stimulation generation coil. Even when the SCR was switched during the discharge operation, there was no voltage or current that exceeded the ratings of the components around the SCR.

Figures 3(a) idea, 3(b) test show the current pulses when S1, S2, S3, and S4 were triggered simultaneously. The delay time was $0 \mathrm{~s}$, and the pulse width was $240 \mu \mathrm{S}$. Figures 4(a) idea, 4(b) test present the current pulses of the stimulation coil after the simultaneous triggering of S1 and S2 and the subsequent triggering of S3 (50 $\mu$ s delay) and S4 (100 $\mu$ s delay). The current pulses had three stages with a pulse width of $720 \mu \mathrm{s}$. Figures 5(a) idea, 5(b) test give the current pulses after triggering S1 at $0 \mathrm{~s}$ without delay, S2 (50 $\mu$ s delay), S3 (100 $\mu$ s delay), and S4 (150 $\mu$ s delay). The pulses had four flat stages with a pulse width of $960 \mu \mathrm{S}$. Figures 6(a) idea, 6(b) test show the current pulses after the simultaneous triggering of S1, S2, and S3 without delay and the triggering of S4 (50 $\mu \mathrm{s}$ delay). They had two high stages with a pulse width of $480 \mu \mathrm{S}$.

Figures 7(a) idea, 7(b) test present the current pulses after triggering $\mathrm{S} 1$ at $0 \mathrm{~s}$ without delay and the simultaneous triggering of S2, S3 (50 $\mu$ s delay), and S4 (100 $\mu$ s delay). The

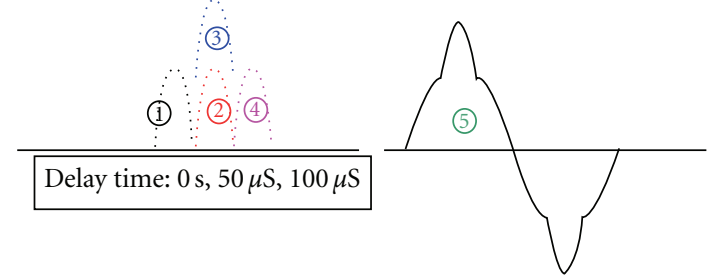

(1) Stimulation pulse by loop-1

(2) Stimulation pulse by loop-2

(3) Stimulation pulse by loop-3

(4) Stimulation pulse by loop-4

(5) Resultant stimulation current pulse

(a)

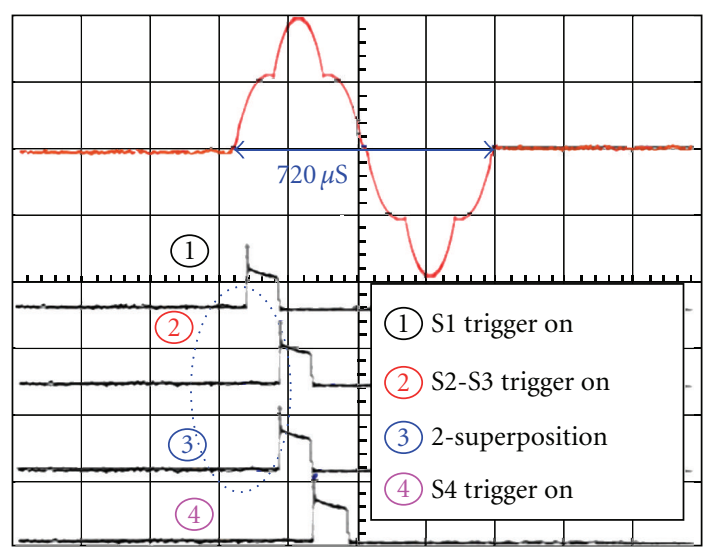

(b)

Figure 7: (a) Idea, (b) test. S1 $\rightarrow$ S2, S3 $\rightarrow$ S4: trigger on (delay: $0 \mathrm{~s}$, $50 \mu \mathrm{S}, 100 \mu \mathrm{S}$; pulse width: $720 \mu \mathrm{S})$.

pulses had three convex stages with a pulse width of $720 \mu \mathrm{S}$. Figures 8(a) idea, $8(\mathrm{~b})$ test give the current pulses after the simultaneous triggering of S1 and S2 and of S3 and S4 (50 $\mu \mathrm{s}$ delay). They had two thick stages with a pulse width of $480 \mu \mathrm{S}$. In Figure 9, the number of triggered SCRs and the delay time led to multistage superposition, which altered the stimulation voltage.

\section{Conclusion}

A multidischarge method was implemented, in which the stimulation coils were driven in sequence via multiple switching control. The limitation of the existing simple sinusoidal pulse form could be overcome by changing the intensity, pulse width, and pulse form.

A new magnetic stimulation device was proposed via multidischarge application, in which diverse treatment current pulses were generated from the stimulation coil by changing the delay for triggering the SCR using an AVR one-chip microprocessor. A wide range of changes in the magnetic field intensity, pulse width, and pulse form control were obtained according to the turn-on time of the stimulation coil and the number of meshes of the PFN. 


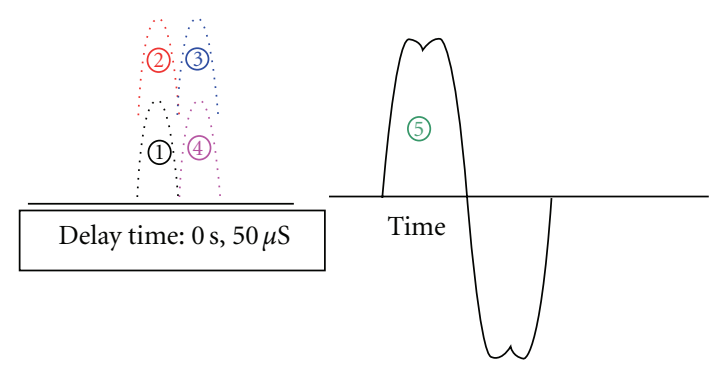

(1) Stimulation pulse by loop-1

(2) Stimulation pulse by loop-2

(3) Stimulation pulse by loop-3

(4) Stimulation pulse by loop-4

(5) Resultant stimulation current pulse

(a)

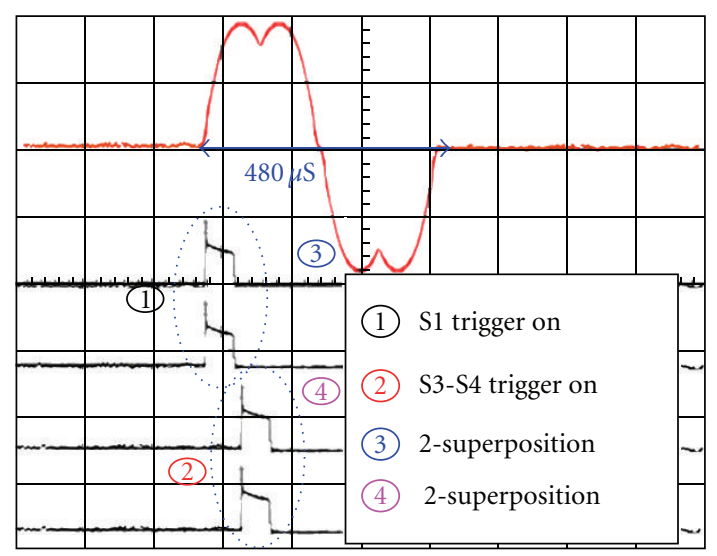

(b)

Figure 8: (a) Idea, (b) test. S1, S2 $\rightarrow$ S3, S4: trigger on (delay: $0 \mathrm{~s}$, $50 \mu \mathrm{S}$; pulse width: $480 \mu \mathrm{S})$.

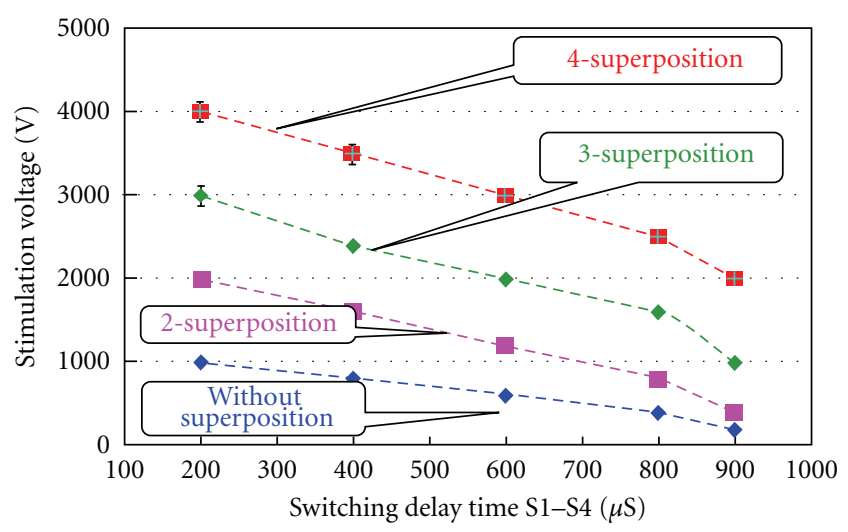

FIGURE 9: Relationship between the superposition and stimulation voltage.

Using AT80S8535 of the ATMEL system, the turn-on delay of the stimulation coil could be controlled in real time within the range of $0 \sim 150 \mu \mathrm{S}$. When the turn-on delay of the stimulation coil, from 0 to $200 \mu \mathrm{S}$, was applied sequentially to superpose the current pulses, the peak of the stimulation coil pulse was $1 \sim 2.7$ times higher than that when the stimulation coil was discharged once, and the pulses were controlled in the pre-, mid-, and postpulse forms. The pulse width was controlled within the range of $240 \sim 960 \mu \mathrm{S}$. The rise and fall times of the pulses can be changed considerably at the first, second, third, and fourth stages.

The one-chip microprocessor technology and PFN were combined to actively superpose the one-four-stage circuits and produce high-voltage pulse forms with diverse pulse widths. Overall, it appears that the limitation of treatments and diagnoses can be overcome by applying a range of pulses to the magnetic stimulation device.

\section{Acknowledgment}

This work was supported by the Dong-A University Research Fund.

\section{References}

[1] M. Inghilleri, A. Berardelli, P. Marchetti, and M. Manfredi, "Effects of diazepam, baclofen and thiopental on the silent period evoked by transcranial magnetic stimulation in humans," Experimental Brain Research, vol. 109, no. 3, pp. 467-472, 1996.

[2] C. Plewnia, M. Bartels, L. Cohen, and C. Gerloff, "Noradrenergic modulation of human cortex excitability by the presynaptic $\alpha$-antagonist yohimbine," Neuroscience Letters, vol. 307 , no. 1 , pp. 41-44, 2001.

[3] S. S. Choi, G. H. Bo, and W. Y. Kim, "Starting current application for magnetic stimulation," Journal of Magnetics, vol. 16, no. 1, pp. 51-57, 2011.

[4] H. Théoret, M. Kobayashi, A. Valero-Cabré, and A. PascualLeone, "Exploring paradoxical functional facilitation with TMS," Supplements to Clinical Neurophysiology, vol. 56, pp. 211-219, 2003.

[5] M. S. George, Transcranial Magnetic Stimulation in Clinical Psychiatry, American Psychiatric Publishing.

[6] E. M. Wassermann, "Risk and safety of repetitive transcranial magnetic stimulation: report and suggested guidelines from the International Workshop on the Safety of Repetitive Transcranial Magnetic Stimulation, June 5-7, 1996," Electroencephalography and Clinical Neurophysiology, vol. 108, no. 1, pp. 1-16, 1998.

[7] L. G. Cohen, B. J. Roth, J. Nilsson et al., "Effects of coil design on delivery of focal magnetic stimulation. Technical considerations," Electroencephalography and Clinical Neurophysiology, vol. 75, no. 4, pp. 350-357, 1990.

[8] V. E. Amassian, R. Q. Cracco, P. J. Maccabee, and J. B. Cracco, "Cerebello-frontal cortical projections in humans studied with the magnetic coil," Electroencephalography and Clinical Neurophysiology, vol. 85, no. 4, pp. 265-272, 1992.

[9] B. W. Wilson, K. Caputa, M. A. Stuchly et al., "Design and fabrication of well confined uniform magnetic field exposure systems," Bioelectromagnetics, vol. 15, no. 6, pp. 563-577, 1994.

[10] S.-S. Choi, S.-M. Lee, and J.-H. Kim, "Chopper application for magnetic stimulation," Journal of Magnetics, vol. 15, no. 4, pp. 213-220, 2010. 

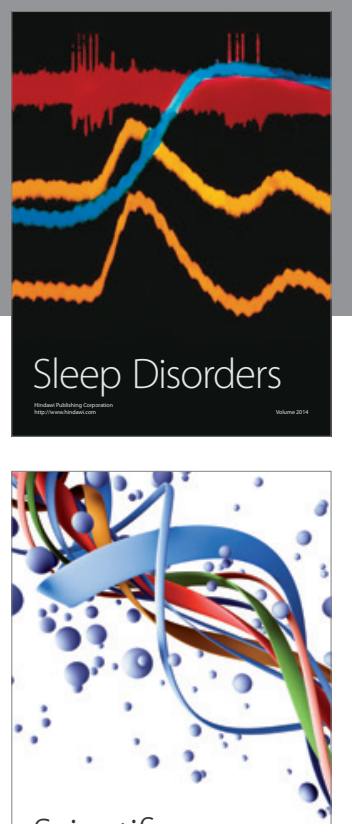

Scientifica
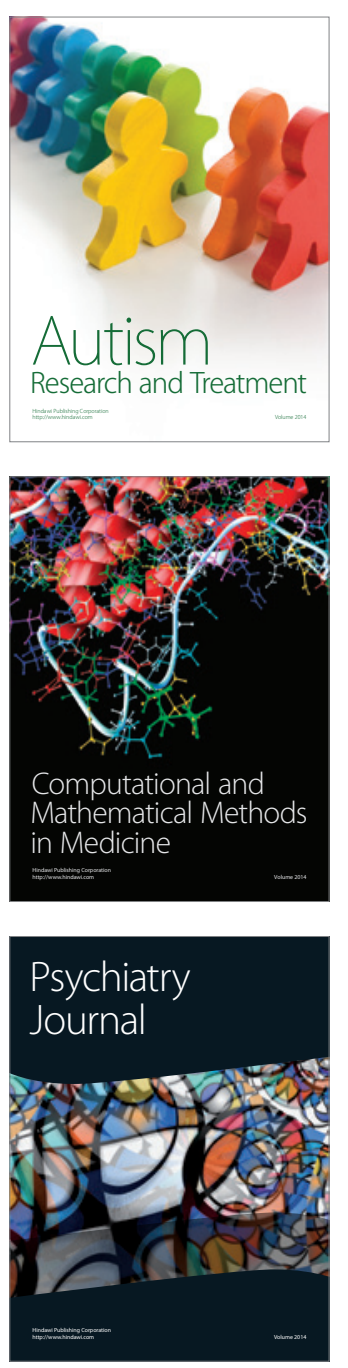
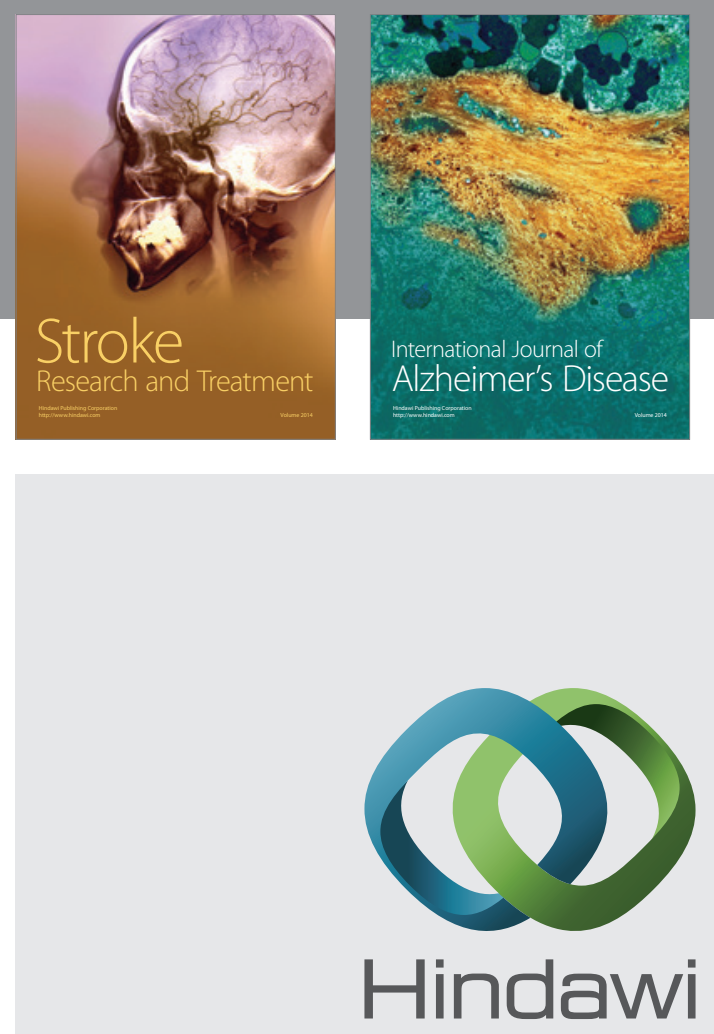

Submit your manuscripts at

http://www.hindawi.com
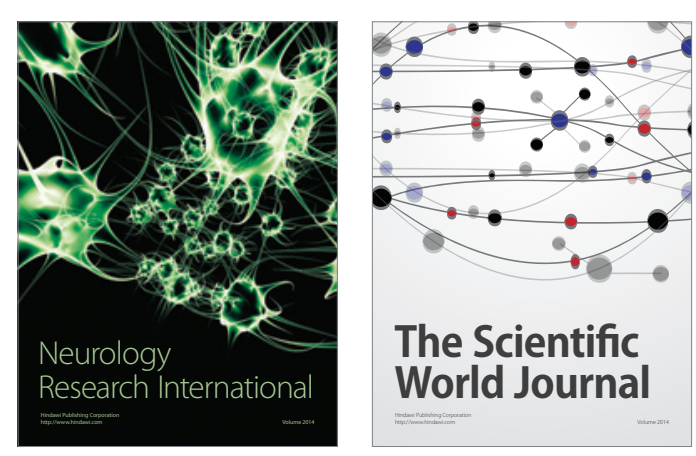

The Scientific World Journal

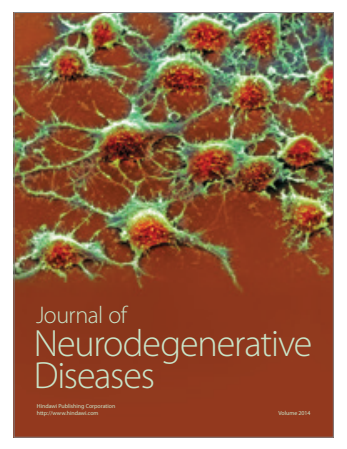

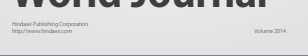

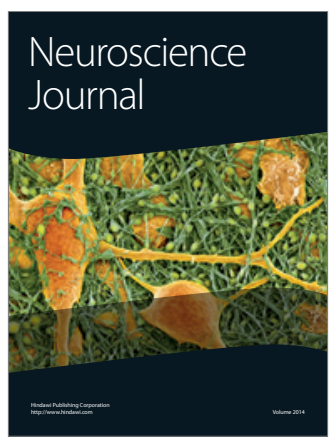

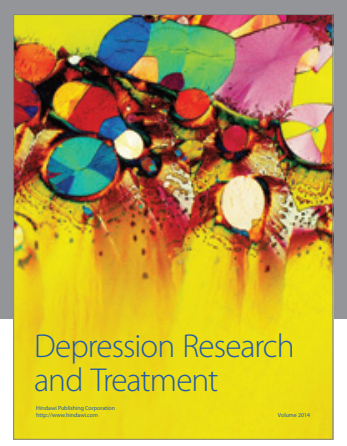
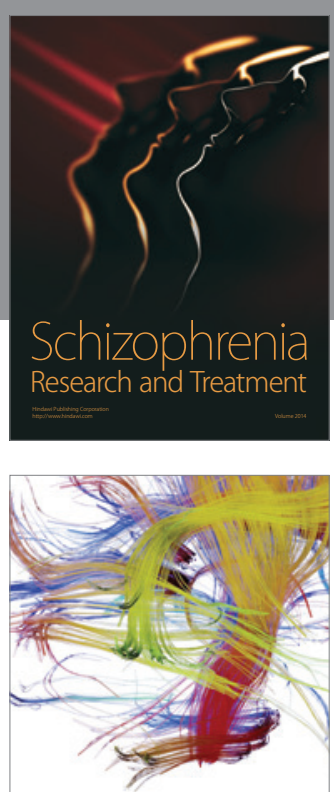

Brain Science

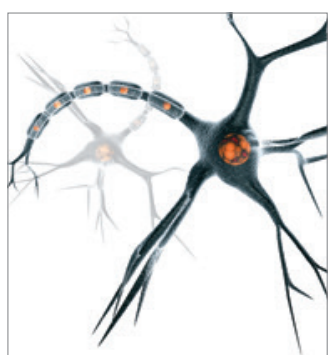

Neural Plasticity
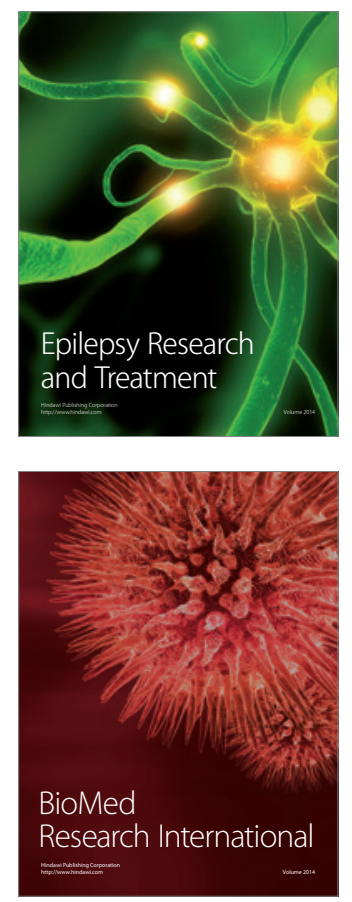

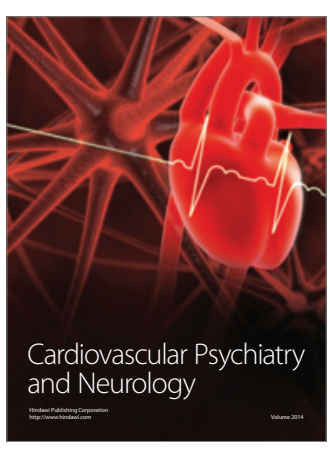

Parkinson's

Disease
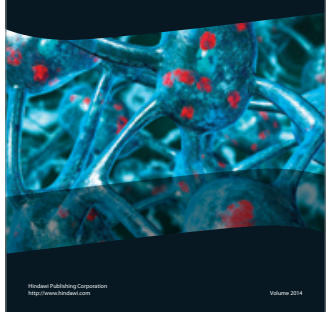